Bulletin of the Section of Logic

Volume 51/2 (2022), pp. 227-242

https://doi.org/10.18778/0138-0680.2021.24

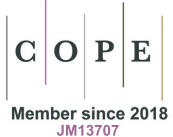

\author{
Alex Belikov \\ Dmitry Zaitsev
}

\title{
A VARIANT OF MATERIAL CONNEXIVE LOGIC
}

\begin{abstract}
The relationship between formal (standard) logic and informal (common-sense, everyday) reasoning has always been a hot topic. In this paper, we propose another possible way to bring it up inspired by connexive logic. Our approach is based on the following presupposition: whatever method of formalizing informal reasoning you choose, there will always be some classically acceptable deductive principles that will have to be abandoned, and some desired schemes of argument that clearly are not classically valid. That way, we start with a new version of connexive logic which validates Boethius' (and thus, Aristotle's) Theses and quashes their converse from right to left. We provide a sound and complete axiomatization of this logic. We also study the implication-negation fragment of this logic supplied with Boolean negation as a second negation.
\end{abstract}

Keywords: Many-valued logics, connexive logic, four-valued logic MC, informal reasoning.

\section{Introduction}

The early 21st century has witnessed a growing interest in connexive logic. One of the reasons for this interest is related to the unsatisfactory formal explication of natural reasoning with logical tools. The point at issue is not only some challengeable classically valid deductive postulates applied as standards of natural reasoning but also of the insufficiency of logical principles accepted in classical logic for an adequate formalization

Presented by: Andrzej Indrzejczak

Received: April 8, 2021

Published online: November 9, 2021

(C) Copyright by Author(s), Łódź 2021

(C) Copyright for this edition by Uniwersytet Łódzki, Łódź 2021 
of reasoning. Our article was prompted by similar considerations: while speculating about the peculiarities of current informal (or argumentative) reasoning formalization, we noted the presence of some undesirable classical deductive principles and the absence of desirable non-classical ones.

In this respect, connexive logic as an alternative to classical logic looks like a very promising candidate. In this paper, we attempt to take another step in this direction and examine some properties of connexive logic that to our way of thinking may be useful for modeling informal, common-sense reasoning.

Connexive logics are usually characterized as requiring that $(A \rightarrow B) \rightarrow$ $(B \rightarrow A)$ is not a theorem, and validating the following set of deductive principles. $^{1}$

- Aristotle's Thesis I: $\neg(A \rightarrow \neg A)$,

- Aristotle's Thesis II: $\neg(\neg A \rightarrow A)$,

- Boethius' Thesis I: $(A \rightarrow \neg B) \rightarrow \neg(A \rightarrow B)$,

- Boethius' Thesis II: $(A \rightarrow B) \rightarrow \neg(A \rightarrow \neg B)$.

The present paper deals, basically, with those connexive logics that fall under the scope of the so-called 'Bochum plan' [8]; that is, being presented in the FDE-like framework (see, for example, [6]) and obtained by tweaking the falsity condition of implication. Plenty of logic were introduced within this paradigm: Wansing's C [18] and MC [19], Omori and Wansing's C3 [16], Omori's dLP [13] and BDW [14], etc. Some related logics that were introduced independently from the Bochum plan, such as Cooper's OL [5, 1], Cantwell's CN [4], and Olkhovikov's LImp [12], nonetheless, can be represented within it. In all these systems, the classically valid converses of Boethius' Theses (BT hereinafter) from right to left are also valid. This made it possible to strengthen the corresponding principles to equivalences:

- Strong Boethius Thesis I: $(A \rightarrow \neg B) \leftrightarrow \neg(A \rightarrow B)$,

- Strong Boethius Thesis II: $(A \rightarrow B) \leftrightarrow \neg(A \rightarrow \neg B)$.

However, this strengthening raises some doubts. For example, S. McCall [10] finds it to be counterintuitive, and H. Wansing and D. Skurt in [20] object to his arguments. The differences of opinion were mainly concerned

\footnotetext{
${ }^{1}$ Notice, however, that there are other ways to characterize connexive logics, see [19]
} 
with the acceptability of BT from right to left in the context of connexive logic, but we argue that these principles clearly are not consistent with the common-sense interpretation. Generally speaking, negating the implication $\neg(A \rightarrow B)$ can mean completely different things. For example in argumentation, there is a distinction between criticism of the claim, or conclusion (rebutting), and criticism of the connection between the reasons and claim (undercutting). In the latter case, $\neg(A \rightarrow B)$ does not mean that $(A \rightarrow \neg B)$. Moreover, this principle can be interpreted as a kind of 'fallacy of relevance': in classical logic, for arbitrary propositional variables $p$ and $q$, it holds that $\neg(p \rightarrow q) \rightarrow(p \rightarrow \neg q)$. It is a kind of a cock and bull story, that might sound like that: 'If it is not true that if elderberry grows in the garden, then my uncle lives in Kyiv, then it is true that if elderberry grows in the garden, then my uncle doesn't live in Kyiv'!

To put it in a nutshell, we believe that the presumptive logic of everyday reasoning should contain some contra-classical deductive principles and not contain some classical ones. We are not alone in this regard. To name but a few, consider recent papers by S. Rahman, H. Rückert et al (consult [17] as a telling example). There is also a self-reliant approach to connexivity associated with experimental philosophy. In particular, paper [7] provides the empirical grounds for various interpretations of the negation of indicative conditionals, which in some cases coincide with the connexive interpretation (see also [15]). In fact, this interpretation of connexivity is based on the same common-sense reasoning that people conduct in the process of arguing. Thus, we incline to believe that concerning implication as a representation of informal (argumentative) reasoning the desirable principles are BT1 and BT2 but not their converses.

On the other hand, this paper is motivated by the question of whether the Bochum plan has sufficient capacity to obtain straightforward and simple semantics that invalidates the converses of BT1 and BT2. And though there is a disagreement about whether it is desirable to have a logic that validates these principles, we believe that all parties could agree that the very opportunity of having a transparent way to obtain a logic without them is certainly desirable. From that perspective, in what follows we will develop our version of connexive logic. In so doing, in the next section, we start with a brief semantical consideration of so-called Minimal Material Connexive logic. Section 3 provides an axiomatization of this logic, and Section 4 deals with the implication-negation fragment of the Minimal Material Connexive logic enriched with Boolean negation. 


\section{Minimal Material Connexive Logic: Semantics}

Throughout the paper we will discuss logics that are built over the standard propositional language $\mathbb{L}$, containing four propositional connectives: implication, conjunction, disjunction, and negation. The notion of a formula is defined in a standard manner. The set of all propositional variables of $\mathbb{L}$ is denoted by $\mathbb{P}$. The set of all formulae of $\mathbb{L}$ is denoted by $\mathbb{F}$.

One of the simplest connexive logics is a four-valued logic MC, introduced by Wansing [18]. It can be seen as an expansion of Dunn-Belnap's logic FDE $[2,3,6]$ with the following implication connective.

\begin{tabular}{c|cccc}
$f_{\mathrm{M} C}$ & $\mathrm{~T}$ & $\mathrm{~B}$ & $\mathrm{~N}$ & $\mathrm{~F}$ \\
\hline $\mathrm{T}$ & $\mathrm{T}$ & $\mathrm{B}$ & $\mathrm{N}$ & $\mathrm{F}$ \\
$\mathrm{B}$ & $\mathrm{T}$ & $\mathrm{B}$ & $\mathrm{N}$ & $\mathrm{F}$ \\
$\mathrm{N}$ & $\mathrm{B}$ & $\mathrm{B}$ & $\mathrm{B}$ & $\mathrm{B}$ \\
$\mathrm{F}$ & $\mathrm{B}$ & $\mathrm{B}$ & $\mathrm{B}$ & $\mathrm{B}$
\end{tabular}

On a par with other related logics, such as Wansing's C [18] and Cantwell's CN [4], MC validates the converses of BT1 and BT2. As we remarked above, this property seems to be counterintuitive within the argumentative context and even criticized by some authors. In what follows, we want to preserve the simplicity of Wansing's approach and obtain a logic where the converses of BT1 and BT2 fail. We denote such logic by MMC which is an abbreviation for 'Minimal Material Connexive Logic'.

We start off by defining the following logical matrix.

Definition 2.1. An MMC-matrix for $\mathbb{L}$ is a tuple $\mathcal{M}=\langle\mathcal{V}, \mathcal{D}, \mathcal{O}\rangle$, where: (a) $\mathcal{V}=\{\mathrm{T}, \mathrm{B}, \mathrm{N}, \mathrm{F}\}$, (b) $\mathcal{D}=\{\mathrm{T}, \mathrm{B}\}$, (c) for every $n$-ary connective $\diamond$ of $\mathbb{L}$, $\mathcal{O}$ contains a corresponding $n$-ary function $f^{\diamond}: \mathcal{V}^{n} \rightarrow \mathcal{V}$. The functions included in $\mathcal{O}$ are defined by means of the following tables:

\begin{tabular}{|c|c|c|c|c|c|c|}
\hline$f^{\urcorner}$ & $A$ & $f \rightarrow$ & $\mathrm{T}$ & B & $\mathrm{N}$ & $F$ \\
\hline $\mathrm{F}$ & $\mathrm{T}$ & $\mathrm{T}$ & $\mathrm{T}$ & B & $F$ & $F$ \\
\hline B & B & B & $\mathrm{T}$ & B & $F$ & $\mathrm{~F}$ \\
\hline $\mathrm{N}$ & $\mathrm{N}$ & $\mathrm{N}$ & B & B & B & B \\
\hline $\mathrm{T}$ & $\mathrm{F}$ & $F$ & B & B & $B$ & B \\
\hline
\end{tabular}




\begin{tabular}{|c|c|c|c|c|c|c|c|c|c|}
\hline$f^{\wedge}$ & $\mathrm{T}$ & B & $\mathrm{N}$ & $\mathrm{F}$ & $f^{\vee}$ & $\mathrm{T}$ & B & $\mathrm{N}$ & $\mathrm{F}$ \\
\hline $\mathrm{T}$ & $\mathrm{T}$ & B & $\mathrm{N}$ & $F$ & $\mathrm{~T}$ & $\mathrm{~T}$ & $\mathrm{~T}$ & $\mathrm{~T}$ & $\mathrm{~T}$ \\
\hline B & B & B & $F$ & $F$ & B & $\mathrm{T}$ & B & $\mathrm{T}$ & B \\
\hline $\mathrm{N}$ & $\mathrm{N}$ & $F$ & $\mathrm{~N}$ & $F$ & $\mathrm{~N}$ & $\mathrm{~T}$ & $\mathrm{~T}$ & $\mathrm{~N}$ & $\mathrm{~N}$ \\
\hline$F$ & $F$ & $F$ & $F$ & $F$ & $F$ & $\mathrm{~T}$ & B & $\mathrm{N}$ & $\mathrm{F}$ \\
\hline
\end{tabular}

An MMC-valuation in an MMC-matrix $\mathcal{M}$ is a function $v: \mathbb{F} \rightarrow \mathcal{V}$ that satisfies the following condition for every $n$-ary connective $\diamond$ of $\mathbb{L}$ and $A_{1}, \ldots, A_{n} \in \mathbb{F}$ :

$$
v\left(\diamond\left(A_{1}, \ldots, A_{n}\right)\right)=f^{\diamond}\left(v\left(A_{1}\right), \ldots, v\left(A_{n}\right)\right) .
$$

Definition 2.2. For every $\Gamma \cup\{A\} \subseteq \mathbb{F}: \Gamma \vDash_{\text {MMC }} A \Leftrightarrow$ for every $v$ in $\mathcal{M}$, if $v(B) \in \mathcal{D}$ (for all $B \in \Gamma$ ), then $v(A) \in \mathcal{D}$. A formula $A$ is called MMC-valid iff $v(A) \in \mathcal{D}$, for every MMC-valuation.

Henceforth, we will use the terms 'matrix', 'valuation', and 'valid' instead of 'MMC-matrix', 'MMC-valuation', and 'MMC-valid', respectively, if otherwise is not required by the context.

Definition 2.3. A generalized truth values MMC-model for $\mathbb{L}$ is a pair $\mathcal{S}=\langle S, \xi\rangle$, where $S$ is the set of generalized truth values $\{\{T, F\},\{T\},\{F\}$, $\varnothing\}$, and $\xi$ is a valuation function mapping every propositional variable into $S$. The following semantic conditions are needed to determine the semantic values of complex formulae.

- $T \in \xi(\neg A) \Leftrightarrow F \in \xi(A)$,

- $F \in \xi(\neg A) \Leftrightarrow T \in \xi(A)$,

- $T \in \xi(A \wedge B) \Leftrightarrow T \in \xi(A)$ and $T \in \xi(B)$,

- $F \in \xi(A \wedge B) \Leftrightarrow F \in \xi(A)$ or $F \in \xi(B)$,

- $F \in \xi(A \vee B) \Leftrightarrow F \in \xi(A)$ and $F \in \xi(B)$,

- $T \in \xi(A \vee B) \Leftrightarrow T \in \xi(A)$ or $T \in \xi(B)$,

- $T \in \xi(A \rightarrow B) \Leftrightarrow$ if $T \in \xi(A)$ then $T \in \xi(B)$,

- $F \in \xi(A \rightarrow B) \Leftrightarrow$ if $T \in \xi(A)$ then $(F \in \xi(B)$ or $T \notin \xi(B))$. 
Definition 2.4. For every $\Gamma \cup\{A\} \subseteq \mathbb{F}: \Gamma \vDash_{\mathcal{S}} A \Leftrightarrow$ for every $\xi$ in $\mathcal{S}$, if $T \in \xi(B)$ (for all $B \in \Gamma$ ), then $T \in \xi(A)$. A formula $A$ is $\mathcal{S}$-valid iff $T \in \xi(A)$ under every valuation in $\mathcal{S}$.

The Wansing-style approach to connexivity is recognized by many authors as the most intuitively plausible. To understand why this is the case, the semantic framework of generalized truth values is very convenient. Indeed, to obtain the semantics for Wansing's MC, it is sufficient to replace the falsity condition of implication from Definition 2.3 with the following one.

$$
F \in \xi(A \rightarrow B) \Leftrightarrow \text { if } T \in \xi(A) \text { then } F \in \xi(B) .
$$

Thus, it is clear that MMC can be considered as a result of the generalization of $\mathbf{M C}$ with respect to the falsity of implication. Intuitively, when the MC-theorists are asserting the falsity of a conditional sentence they are quite opinionated regarding the epistemic status of the consequent. In turn, MMC-theorists are more 'non-deterministic' in this respect.

We finish this section by proving the equivalence of the matrix and generalized truth values semantics of MMC.

Lemma 2.5. For every $A \in \mathbb{F}$, every MMC-valuation $v$ in an MMCmatrix $\mathcal{M}$, and every $\mathcal{S}$-valuation in a generalized truth values $\mathbf{M M C}$ model $\mathcal{S}$ the following hold.

$$
\begin{aligned}
& v(A)=\mathrm{T} \Leftrightarrow T \in \xi(A) \text { and } F \notin \xi(A), \\
& v(A)=\mathrm{B} \Leftrightarrow T \in \xi(A) \text { and } F \in \xi(A), \\
& v(A)=\mathrm{N} \Leftrightarrow T \notin \xi(A) \text { and } F \notin \xi(A), \\
& v(A)=\mathrm{F} \Leftrightarrow T \notin \xi(A) \text { and } F \in \xi(A) .
\end{aligned}
$$

Proof: Using standard induction on the complexity of $A$.

With the help of Lemma 2.5, the following theorem can be proven.

Theorem 2.6. For any $\Gamma \cup\{A\} \subseteq \mathbb{F}: \Gamma \vDash_{\text {MMC }} A \Leftrightarrow \Gamma \vDash_{\mathcal{S}} A$.

Proof: Assume $\Gamma \nvdash_{\text {MMC }} A$. Then, by Definition 2.2, there is an MMCvaluation $v$ in a MMC-matrix $\mathcal{M}$, such that $v(\Gamma) \subseteq\{\mathrm{T}, \mathrm{B}\}$ and $v(A) \nsubseteq$ $\{\mathrm{T}, \mathrm{B}\}$, that is $v(A) \subseteq\{\mathrm{N}, \mathrm{F}\}$. By Lemma 2.5, there is an $\mathcal{S}$-valuation $\xi$ in a generalized truth values MMC-model $\mathcal{S}$, such that $T \in \xi(B)$ for all $B \in \Gamma$ and $T \notin \xi(A)$, thereby providing $\Gamma \not_{\mathcal{S}} A$ (by Definition 2.4). Therefore, 
if $\Gamma \vDash_{\mathcal{S}} A$, then $\Gamma \vDash_{\text {MMC }} A$. The converse of the latter statement can be proven by analogous argument.

We consider it acceptable to designate our logic by the term 'minimal' for the following reason. In [9] Estrada-González and Ramirez-Cámara suggested a list of desiderata that can be used to distinguish between different senses of connexivity. According to their list, a logic is claimed to be minimally connexive if it validates both Aristotle theses, BT1, BT2 and invalidates $(A \rightarrow B) \rightarrow(B \rightarrow A)$, i.e. exactly the same requirements that we discussed in the introduction section. Again, according to [9], a logic is called 'subminimal connexive' if it satisfies at least some but not all of the just mentioned conditions. The logic MC can be rightfully claimed as 'minimal' in Estrada-González and Ramirez-Cámara sense. However, one could use another notion of minimality by requiring in addition that the converses of Boethius' theses fail to be valid. In this different sense of minimality MMC is more suitable than MC. For counterexamples falsifying Boethius' theses in MMC, consider a valuation $v$, such that $v(A)=\mathrm{T}$ and $v(B)=\mathrm{N}$. Thus, MMC is a kind of a 'downgraded' version of MC.

Finally, both logics are inconsistent but not trivial, as $(p \wedge \neg p) \rightarrow p$ and $\neg((p \wedge \neg p) \rightarrow p)$ are simultaneously valid in MMC and MC.

\section{Minimal Material Connexive Logic: Axiomatization}

\subsection{Proof-theory}

A Hilbert-style proof-system $\mathcal{H}$ for $\mathbf{M M C}$ is defined by means of the following axioms and rules.

Axioms:

(A1) $A \rightarrow(B \rightarrow A)$,

(A2) $(A \rightarrow(B \rightarrow C)) \rightarrow((A \rightarrow B) \rightarrow(A \rightarrow C))$,

(A3) $((A \rightarrow B) \rightarrow A) \rightarrow A$,

(A4) $((A \rightarrow C) \wedge(B \rightarrow C)) \rightarrow((A \vee B) \rightarrow C)$,

(A5) $A \rightarrow(A \vee B)$, 
(A6) $B \rightarrow(A \vee B)$,

(A7) $((A \rightarrow B) \wedge(A \rightarrow C)) \rightarrow(A \rightarrow(B \wedge C))$,

(A8) $(A \wedge B) \rightarrow A$,

(A9) $(A \wedge B) \rightarrow B$,

(A10) $\neg \neg A \leftrightarrow A$,

(A11) $\neg(A \wedge B) \leftrightarrow(\neg A \vee \neg B)$,

$(\mathrm{A} 12) \neg(A \vee B) \leftrightarrow(\neg A \wedge \neg B)$,

(A13) $(A \rightarrow \neg B) \rightarrow \neg(A \rightarrow B)$,

(A14) $B \rightarrow(\neg(A \rightarrow B) \rightarrow(A \rightarrow \neg B))$,

(A15) $A \vee \neg(B \rightarrow A)$,

INFERENCE RULES:

(MP) $\frac{A \rightarrow B, \quad A}{B}$.

Definition 3.1. A proof of a formula $A$ in $\mathcal{H}$ is a sequence of formulas $A_{1}, \ldots, A_{n}, A$, where $0 \leq n$, such that every formula in the sequence $A_{1}, \ldots, A_{n}, A$ either ( 1$)$ is an axiom of $\mathcal{H}$, or (2) is obtained with the help of (MP) from the preceding formulas.

We write $\vdash_{\mathcal{H}} A$ to denote that $A$ has a proof in $\mathcal{H}$ (such $A$ is called a theorem). $\Gamma \vdash_{\mathcal{H}} A$ means that $A$ has a proof from hypotheses $\Gamma$ in $\mathcal{H}$, i. e. there is a sequence of formulas $A_{1}, \ldots, A_{n}, A$, where $0 \leq n$, such that every formula in the sequence $A_{1}, \ldots, A_{n}, A$ either (1) belongs to $\Gamma$, or (2) is an axiom of $\mathcal{H}$, or (3) is obtained with the help of (MP) from the preceding formulas. As in the case of MC, the pure implicational fragment of MMC is classical, hence the following Deduction Theorem is provable in MMC. 
Theorem 3.2. For any $\Gamma \cup\{A, B\} \subseteq \mathbb{F}$, if $\Gamma, A \vdash_{\mathcal{H}} B$, then $\Gamma \vdash_{\mathcal{H}} A \rightarrow B$. Proof: The proof is quite standard, it can be obtained using (A1), (A2) and (MP). For details the reader can consult, for example, [11].

Interestingly, a Hilbert-style system for Wansing's MC can be obtained by making a simple change to $\mathcal{H}$, namely it is sufficient to replace (A14) and (A15) with

$$
\neg(A \rightarrow B) \rightarrow(A \rightarrow \neg B) .
$$

Notice, however, that (A14) should not be disregarded by MC-theorists because it is, in fact, a theorem of $\mathbf{M C}$ and can be proven, using (A1) and (CBT 1).

Nevertheless, $\mathbf{M C}$ is not an extension of $\mathbf{M M C}$ since (A15) is not a theorem of MC. This can be easily checked with the help of the soundness result for MC. Apropos it establishes the independence of (A15) in MMC. Thus, both logics are orthogonal to each other.

\subsection{Completeness and soundness of MMC}

Now we turn to the completeness proof and begin with some auxiliary notions.

Definition 3.3. A set of formulas $\mathcal{T}$ is called a theory if it is closed under $\vdash_{\mathcal{H}}$. A theory $\mathcal{T}$ is called prime iff, for every formulas $A$ and $B$, it holds that $A \vee B \in \mathcal{T}$ implies $A \in \mathcal{T}$ or $B \in \mathcal{T}$. Finally, $\mathcal{T}$ is called non-trivial if $A \notin \mathcal{T}$ for some formula $A$.

To pave the way for the proof of the canonical valuation lemma, consider the following set of derivable formulae.

FACT 3.4. The formulae below are derivable.

$$
\begin{gathered}
A \vee(A \rightarrow B) \\
A \vee \neg(A \rightarrow B) \\
\neg B \rightarrow \neg(A \rightarrow B)
\end{gathered}
$$

Proof: For (T1) we use (A3) and Theorem 3.2. For (T2) we use (T1) and (A13). For (T3) we use (A1) and (A13).

Now is the time to prove the following useful lemma. 
Lemma 3.5. For any prime theory $\Gamma$, it holds that $A \rightarrow B \in \Gamma$ iff $A \notin \Gamma$ or $B \in \Gamma$.

Proof: Suppose $A \rightarrow B \in \Gamma$ and $A \in \Gamma$ and $B \notin \Gamma$. Then, by (MP), we have a contradiction. For the converse we have two cases. Suppose that $A \notin \Gamma$ and $A \rightarrow B \notin \Gamma$. Then, by (T1), we have contradiction, since $\Gamma$ is prime. Now, suppose that $B \in \Gamma$ and $A \rightarrow B \notin \Gamma$. Then, using (A1) and (MP), we obtain contradiction.

DeFinition 3.6. Let $\mathcal{T}$ be a non-empty non-trivial prime theory. We define an MMC-canonical valuation $\xi_{\mathcal{T}}$ by requiring that for every $p \in \mathbb{P}$ :

$$
T \in \xi_{\mathcal{T}}(p) \Leftrightarrow p \in \mathcal{T}, \quad F \in \xi_{\mathcal{T}}(p) \Leftrightarrow \neg p \in \mathcal{T} .
$$

Lemma 3.7. Let $\xi_{\mathcal{T}}$ be a MMC-canonical valuation. Then, for every $A \in \mathbb{F}$, the following holds.

$$
T \in \xi_{\mathcal{T}}(A) \Leftrightarrow A \in \mathcal{T}, \quad F \in \xi_{\mathcal{T}}(A) \Leftrightarrow \neg A \in \mathcal{T} .
$$

Proof: By induction on the complexity of a formula $A$. We abbreviate 'inductive hypothesis' by 'IH'. The basic case, when $A \in \mathbb{P}$, follows from Definition 3.6.

For negation. We begin with the truth condition: $T \in \xi_{\mathcal{T}}(\neg B)$ iff $F \in$ $\xi_{\mathcal{T}}(B)$ (by Definition 2.3) iff $\neg B \in \mathcal{T}$ (by IH). The falsity condition is also straightforward: $F \in \xi_{\mathcal{T}}(\neg B)$ iff $T \in \xi_{\mathcal{T}}(B)$ iff $B \in \mathcal{T}$ (by IH) iff $\neg \neg B \in \mathcal{T}$ (by $(\mathrm{A} 10)$ ).

For conjunction. $T \in \xi_{\mathcal{T}}(B \wedge C)$ iff $T \in \xi_{\mathcal{T}}(B)$ and $T \in \xi_{\mathcal{T}}(C)$ (by Definition 2.3) iff $B \in \mathcal{T}$ and $C \in \mathcal{T}$ (by IH) iff $B \wedge C \in \mathcal{T}$ (by (A7), (A8), (A9)). $F \in \xi_{\mathcal{T}}(B \wedge C)$ iff $F \in \xi_{\mathcal{T}}(B)$ or $F \in \xi_{\mathcal{T}}(C)$ (by Definition 2.3) iff $\neg B \in \mathcal{T}$ or $\neg C \in \mathcal{T}$ (by IH) iff $\neg(B \wedge C) \in \mathcal{T}$ (by (A11)).

For disjunction. $T \in \xi_{\mathcal{T}}(B \vee C)$ iff $T \in \xi_{\mathcal{T}}(B)$ or $T \in \xi_{\mathcal{T}}(C)$ (by Definition 2.3) iff $B \in \mathcal{T}$ or $C \in \mathcal{T}$ (by IH) iff $B \vee C \in \mathcal{T}$ (by (A5), (A6) and primeness). $F \in \xi_{\mathcal{T}}(B \vee C)$ iff $F \in \xi_{\mathcal{T}}(B)$ and $F \in \xi_{\mathcal{T}}(C)$ (by Definition 2.3) iff $\neg B \in \mathcal{T}$ and $\neg C \in \mathcal{T}$ (by IH) iff $\neg(B \vee C) \in \mathcal{T}$ (by (A12)).

For implication. Let $F \in \xi_{\mathcal{T}}(B \rightarrow C)$ and $\neg(B \rightarrow C) \notin \mathcal{T}$. Then, by Definition 2.3, we have 


$$
T \notin \xi_{\mathcal{T}}(B) \text { or }\left(F \in \xi_{\mathcal{T}}(C) \text { or } T \notin \xi_{\mathcal{T}}(C)\right) .
$$

There are the three cases to consider.

$$
\begin{array}{llll}
\text { (a) } T \notin \xi_{\mathcal{T}}(B) & \Rightarrow_{\mathrm{IH}} & B \notin \mathcal{T} \\
\text { (b) } F \in \xi_{\mathcal{T}}(C) & \Rightarrow_{\mathrm{IH}} & \neg C \in \mathcal{T}, \\
\text { (c) } T \notin \xi_{\mathcal{T}}(C) & \Rightarrow_{\mathrm{IH}} & C \notin \mathcal{T} .
\end{array}
$$

We have to show that every case leads to a contradiction. For (a) we use $B \vee \neg(B \rightarrow C)$ (see (T2), Fact 3.4) and primeness of $\mathcal{T}$. For (b) we use $\neg C \rightarrow \neg(B \rightarrow C)$ (see (T3), Fact 3.4). The proof of (c) uses $C \vee \neg(B \rightarrow C) \in \mathcal{T}$, which is provable with the help of (A15).

Suppose $\neg(B \rightarrow C) \in \mathcal{T}$ and $F \notin \xi_{\mathcal{T}}(B \rightarrow C)$. From the latter, using Definition 2.3, we obtain

$$
T \in \xi_{\mathcal{T}}(B) \text { and } F \notin \xi_{\mathcal{T}}(C) \text { and } T \in \xi_{\mathcal{T}}(C) .
$$

By IH,

$$
B \in \mathcal{T} \text { and } \neg C \notin \mathcal{T} \text { and } C \in \mathcal{T} .
$$

From this, using $C \rightarrow(\neg(B \rightarrow C) \rightarrow(B \rightarrow \neg C))$ (A14), we obtain a contradiction. Therefore, $F \in \xi_{\mathcal{T}}(B \rightarrow C)$.

Let $T \in \xi_{\mathcal{T}}(B \rightarrow C)$. Then, by Definition 2.3, $T \notin \xi_{\mathcal{T}}(B)$ or $T \in \xi_{\mathcal{T}}(C)$. Applying IH, we have $B \notin \mathcal{T}$ or $C \in \mathcal{T}$. Now Lemma 3.5 gives the desired result in both directions.

We use a standard version of Lindenbaum's lemma, so its proof is omitted.

Lemma 3.8. For any $\Gamma \cup\{A\} \subseteq \mathbb{F}$, if $\Gamma \forall_{\mathcal{H}} A$, then, there exists a nontrivial prime theory $\Gamma^{\prime}$, such that $\Gamma \subseteq \Gamma^{\prime}$ and $\Gamma^{\prime} \forall_{\mathcal{H}} A$.

Thus, we can move toward the completeness theorem.

Theorem 3.9. For any $\Gamma \cup\{A\} \subseteq \mathbb{F}$, if $\Gamma \vDash_{\mathcal{S}} A$, then $\Gamma \vdash_{\mathcal{H}} A$.

Proof: As usual.

Finally, the soundness theorem is proven as usual. 
Theorem 3.10. For any $\Gamma \cup\{A\} \subseteq \mathbb{F}$, if $\Gamma \vdash_{\mathcal{H}} A$, then $\Gamma \vdash_{\mathcal{S}} A$.

Proof: As usual.

In virtue of Theorem 2.6 we obtain the following corollary.

Corollary 3.11. For any $\Gamma \cup\{A\} \subseteq \mathbb{F}, \Gamma \vdash_{\mathcal{H}} A$ iff $\Gamma \vDash_{\text {MMC }} A$.

\section{Expanding pure connexive fragment of MMC with Boolean negation}

Slightly adopting the terminology from [21] we will denote the implicationnegation fragment of MMC as 'pure connexive fragment'. Moving further along this avenue we arrive at relatively poor logic with implication and negation as the only primitives. In fact, in this case, it is interesting to add the second Boolean negation $\sim$ to indicate non relevant arguments, so that $\sim A$ means that $A$ is absent from the current argumentative discourse. This move will allow comparing the behavior of two negations in the same context and at the same time will simplify the axiomatization of the resulting fragment.

Boolean negation differs from DeMorgan $\neg$ only in $\mathrm{N}$ and $\mathrm{B}$ lines: $\sim \mathrm{N}=$ $\mathrm{B}, \sim \mathrm{B}=\mathrm{N}$. Thus, we add to Definition 3 two items:

$$
\begin{aligned}
& T \in \xi(\sim A) \Leftrightarrow T \notin \xi(A) \\
& F \in \xi(\sim A) \Leftrightarrow F \notin \xi(A)
\end{aligned}
$$

The resulting system will be implication-negation fragment of MMC supplied with an extra negation: $\mathbf{M M C} \stackrel{\neg, \sim}{\longrightarrow}$. A Hilbert-style axiomatization of $\mathbf{M M C} \stackrel{\neg, \sim}{\longrightarrow}$ contains (MP), (A1) - (A2), (A10), (A13), (A14), and the following list of axioms:

$(\mathrm{A} 16)(\sim C \rightarrow \sim B) \rightarrow((\sim C \rightarrow B) \rightarrow C)$,

(A17) $\sim A \leftrightarrow \neg \sim A$,

(A18) $(A \rightarrow \sim B) \rightarrow \neg(A \rightarrow B)$,

The completeness proof becomes simpler. Now we do not need theories to be prime, instead we require them to be normal with respect to the Boolean negation: $\sim A \in \mathcal{T} \Leftrightarrow A \notin \mathcal{T}$. Moreover, we impose the following require-

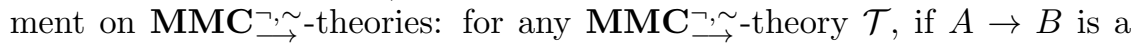


theorem of $\mathbf{M M C} \stackrel{\neg, \sim}{\longrightarrow}$ and $A \in \mathcal{T}$, then $B \in \mathcal{T}$. Taking these modifications into account, the definition of canonical valuation is otherwise preserved. We no longer need Lemma 2, and can immediately proceed to the proof of an analogue of Lemma 3. Consider only the different cases.

For Boolean negation. We begin with the truth condition: $T \in \xi_{\mathcal{T}}(\sim B)$ iff $T \notin \xi_{\mathcal{T}}(B)$ (by (BN1)) iff $B \notin \mathcal{T}$ (by IH) iff $\sim B \in \mathcal{T}$ (by normality). The falsity condition is also straightforward: $F \in \xi_{\mathcal{T}}(\sim B)$ iff $F \notin \xi_{\mathcal{T}}(B)$ iff $\neg B \notin \mathcal{T}$ (by IH) iff $\sim \neg B \in \mathcal{T}$ (by normality) iff $\neg \sim B \in \mathcal{T}$ (by (A17)).

For implication. When $T \in \xi_{\mathcal{T}}(B \rightarrow C)$, there are two cases: $T \notin \xi_{\mathcal{T}}(B)$ or $T \in \xi_{\mathcal{T}}(C)$. By IH, they may be presented as $C \in \mathcal{T}$ or $B \notin \mathcal{T}$. The first option exploits (A1) while the proof in the second case is based on (T4) $\sim B \rightarrow(B \rightarrow C)$ : using the normality of $\mathcal{T}$, we obtain $\sim B \in \mathcal{T}$, and hence, by (T4), we have $B \rightarrow C \in \mathcal{T}$.

The case with $F \in \xi_{\mathcal{T}}(B \rightarrow C)$ falls into three possibilities:
(a) $T \notin \xi_{\mathcal{T}}(B) \quad \Rightarrow_{\mathrm{IH}} \quad B \notin \mathcal{T}$
(b) $F \in \xi_{\mathcal{T}}(C) \quad \Rightarrow_{\mathrm{IH}} \quad \neg C \in \mathcal{T}$,
(c) $T \notin \xi_{\mathcal{T}}(C) \quad \Rightarrow_{\mathrm{IH}} \quad C \notin \mathcal{T}$.

For (a) (T4) is needed to show that $\sim B \rightarrow \neg(B \rightarrow C)$, for (b) apply substitutional case of (A1), and (c) requires (A18) to receive $\sim C \rightarrow \neg(B \rightarrow C)$.

Lindenbaum's Lemma is proved in a natural way.

\section{Conclusion}

In this work, we were concerned with the problem of modifying a fourvalued connexive implication in such a way that allows it to fit better in the context of common-sense reasoning. As far as we can see, the resulting implication, aside from the ultimate goal, allows one to use a connexive logic with another (more restrictive) notion of minimality, compared to the implication of Wansing's logic MC. We equipped the corresponding logic MMC with a sound and complete Hilbert-style calculus. We also considered implication-negation fragment of $\mathbf{M M C}$, expanded with the 
Boolean negation and provided an adequate Hilbert-style calculus for this fragment. As to the future work, we find it interesting to explore how the ideas presented in this paper can be applied to other Wansing-style connexive logics, especially to constructive connexive logics.

Acknowledgements. This research is supported by Russian Science Foundation (project №20-18-00158).

\section{References}

[1] A. Belikov, Peirce's Triadic Logic and Its (Overlooked) Connexive Expansion, Logic and Logical Philosophy, vol. 30(3) (2021), pp. 535-559, DOI: https://doi.org/10.12775/LLP.2021.007.

[2] N. D. Belnap, A Useful Four-Valued Logic, [in:] J. M. Dunn, G. Epstein (eds.), Modern Uses of Multiple-Valued Logic, vol. 2 of Episteme (A Series in the Foundational, Methodological, Philosophical, Psychological, Sociological, and Political Aspects of the Sciences, Pure and Applied), Springer Netherlands, Dordrecht (1977), pp. 5-37, DOI: https://doi.org/10.1007/978-94-010-1161-7_2.

[3] N. D. Belnap, How a Computer Should Think, [in:] H. Omori, H. Wansing (eds.), New Essays on Belnap-Dunn Logic, Springer International Publishing, Cham (2019), pp. 35-53, DOI: https://doi.org/10.1007/978-3-03031136-0_4.

[4] J. Cantwell, The Logic of Conditional Negation, Notre Dame Journal of Formal Logic, vol. 49(3) (2008), pp. 245-260, DOI: https://doi.org/10. 1215/00294527-2008-010.

[5] W. Cooper, The Propositional Logic of Ordinary Discourse, Inquiry, vol. 11(1-4) (1968), pp. 295-320, DOI: https://doi.org/10.1080/ 00201746808601531.

[6] J. M. Dunn, Intuitive semantics for first-degree entailments and 'coupled trees', Philosophical Studies, vol. 29(3) (1976), pp. 149-168, DOI: https: //doi.org/10.1007/BF00373152.

[7] P. Egré, G. Politzer, On the negation of indicative conditionals, [in:] M. Aloni, M. Franke, F. Roelofsen (eds.), Proceedings of the 19th Amsterdam Colloquium (2013), pp. 10-18, URL: http://events.illc.uva.nl/ AC/AC2013/uploaded_files/inlineitem/02_Egre_Politzer.pdf. 
[8] L. Estrada-González, The Bochum Plan and the foundations of contraclassical logics, CLE e-Prints, vol. 19(1) (2020), pp. 1-22.

[9] L. Estrada-González, E. Ramirez-Cámara, A Comparison of Connexive Logics, IfCoLog Journal of Logics and their Applications, vol. 3 (2016), pp. 341-355.

[10] S. McCall, A History of Connexivity, [in:] D. Gabbay (ed.), Handbook of the History of Logic, vol. 11, Elsevier (2012), pp. 415-449.

[11] E. Mendelson, Introduction to Mathematical Logic (1987), DOI: https://doi. org/10.1007/978-1-4615-7288-6.

[12] G. Olkhovikov, On a New Three-Valued Paraconsistent Logic, [in:] Logic of Law and Tolerance, Ural University Press (2002), pp. 96-113.

[13] H. Omori, From Paraconsistent Logic to Dialetheic Logic, [in:] H. Andreas, P. Verdée (eds.), Logical Studies of Paraconsistent Reasoning in Science and Mathematics, vol. 45 of Trends in Logic (Studia Logica Library), Springer (2016), pp. 111-134.

[14] H. Omori, A Simple Connexive Extension of the Basic Relevant Logic BD, IfCoLog Journal of Logics and their Applications, vol. 3(3) (2016), pp. $467-478$.

[15] H. Omori, Towards a bridge over two approaches in connexive logic, Logic and Logical Philosophy, vol. 28(3) (2019), pp. 553-556, DOI: https:// doi.org/10.12775/llp.2019.005.

[16] H. Omori, H. Wansing, An Extension of Connexive Logic C, [in:] N. Olivietti, R. Verbrugge, S. Negri, G. Sandu (eds.), Advances in Modal Logic, vol. 13, College Publications, Rickmansworth (2020), pp. 503-522.

[17] S. Rahman, On Hypothetical Judgements and Leibniz's Notion of Conditional Right, [in:] M. Armgardt, P. Canivez, S. Chassagnard-Pinet (eds.), Past and Present Interactions in Legal Reasoning and Logic, Springer International Publishing, Cham (2015), pp. 109-167, DOI: https://doi.org/10.1007/978-3-319-16021-4_7.

[18] H. Wansing, Connexive Modal Logic, [in:] R. Schmidt (ed.), Advances in Modal Logic, vol. 5, College Publications (2005), pp. 367-383.

[19] H. Wansing, Connexive Logic, [in:] E. N. Zalta (ed.), The Stanford Encyclopedia of Philosophy, spring 2021 ed., Metaphysics Research Lab, Stanford University (2021), URL: https://plato.stanford.edu/archives/spr2021/ entries/logic-connexive/. 
[20] H. Wansing, D. Skurt, Negation as Cancellation, Connexive Logic, and $q L P m$, The Australasian Journal of Logic, vol. 15(2) (2018), pp. 476488, DOI: https://doi.org/10.26686/ajl.v15i2.4869.

[21] Y. Weiss, Semantics For Pure Theories of Connexive Implication, The Review of Symbolic Logic, (2020), pp. 1-16, DOI: https://doi.org/ 10.1017/S1755020320000374.

\author{
Alex Belikov \\ Lomonosov Moscow State University \\ Department of Logic, Faculty of Philosophy \\ 119234, Lomonosovskij prospekt, 27/4 \\ Moscow, Russian Federation \\ e-mail: belikov@philos.msu.ru \\ Dmitry Zaitsev \\ Lomonosov Moscow State University \\ Department of Logic, Faculty of Philosophy \\ 119234, Lomonosovskij prospekt, 27/4 \\ Moscow, Russian Federation \\ e-mail: zaitsev@philos.msu.ru
}

